\title{
What the Brain can Tell us in Musculoskeletal Rehabilitation
}

\section{Jochen Baumeister*}

Exercise \& Brain Laboratory, Institute of Sports Medicine, University of Paderborn, Germany

Research in musculoskeletal rehabilitation has grown tremendously in the past few decades. Most of this research is based on a biomechanical approach demonstrating peripheral differences between patients and controls in kinetic, kinematic and electromyographic data. The scientific community describes motor behavior as the final common output of the human sensorimotor system which provides valuable insights into motor changes after musculoskeletal injuries and damages.

But the sensorimotor system covers far more than bones and muscles to provide control of motor behavior. Sensorimotor control describes the dynamic interaction between sensations of sensory stimuli (e.g. proprioceptive, visual, vestibular), the processing and integration of these information in the CNS and the biomechanically well described motor output [1].

Undoubtedly the brain is the supervisor of sensorimotor control. It is important to know how the brain regulates the control mechanisms in order to understand how training in rehabilitation should be designed to compensate in motor deficits after injury [2]. Only this brain-behavior relationship can provide the foundation of rehabilitation programs.

The ACL injury seems to be the most investigated musculoskeletal injury and therefore is a good model in this case. There had been extensive research to highlight the deficits like muscle strength [3], postural control [4] and coordination patterns in functional performance [5-7] which occur after ACL injury and/or reconstruction. These deficiencies are thought to be related to the disturbance of the sensorimotor control process $[8,9]$. On a theoretical basis different authors hypothesize that the deficiency in motor output is related to the change of the reception of afferent proprioceptive information due to receptor damage in the knee caused by ACL injury or reconstruction, which most likely lead to modifications in the CNS and is not simply based on a mechanical instability [10].

The progress in neuroscience opens new possibilities to take a look into brain mechanisms in musculoskeletal rehabilitation. Functional MRI (fMRI) and electroencephalography (EEG) are able to provide data due to brain activity and connectivity $[11,12]$ in a relationship to ACL deficiency. Recently a few researchers followed these research line and started exploratory pioneer work to add measures of brain activity in sensorimotor control after ACL injury. They detected brain areas like the frontal and parietal cortical areas related to higher executive functions, somatosensory information processing and the cerebellum due to fine-tuning in sensorimotor control which serve as a neuronal correlate to motor behavior [13-15]. It is hypothesized that the working memory as a mechanism for integration of relevant information into the movement planning may play specific a role after ACL injury [13]. Changed afferent feedback is thought to increase executive attentional control and processing activity in the somatosensory areas. These results attract new methodological approaches to look into the frontoparietal network communication inside the brain and into brainmuscle communication by EEG functional connectivity measures [16] in ACL research. But even if the results are promising: we are still at the beginning to discover brain mechanisms after musculoskeletal injuries, but it is a start!

For future research in sensorimotor control related musculoskeletal rehabilitation this example should demonstrate the importance to open the window to the brain related to motor behavior. Only the "synchronized" look at the internal processing and the external motor output can help to discover the underlying mechanisms behind injury and damage. Neuroscience and biomechanics should work closely together in an interdisciplinary approach to reach the final goal in musculoskeletal rehabilitation research: to build a scientific base for optimized rehabilitation programs with the understanding why the programs work which will be highly beneficial for the patient.

\section{References}

1. Baumeister J, Reinecke K, Schubert M, Schade J, Weiss M (2011) Effects of induced fatigue on brain activity during sensorimotor control. Eur J Appl Physiol.

2. Powers CM, Fisher B (2010) Mechanisms underlying ACL injury-prevention training: the brain-behavior relationship. J Athl Train 45: 513-515.

3. Xergia SA, McClelland JA, Kvist J, Vasiliadis HS, Georgoulis AD (2011) The influence of graft choice on isokinetic muscle strength 4-24 months after anterior cruciate ligament reconstruction. Knee Surg Sports Traumatol Arthrosc 19: $768-780$

4. Ageberg E, Roberts D, Holmstrom E, Friden T (2005) Balance in single-limb stance in patients with anterior cruciate ligament injury: relation to knee laxity, proprioception, muscle strength, and subjective function. Am J Sports Med. Am J Sports Med 33: 1527-1535.

5. Katayama M, Higuchi H, Kimura M, Kobayashi A, Hatayama K, et al. (2004) Proprioception and performance after anterior cruciate ligament rupture. Int Orthop 28: 278-281.

6. Yamazaki J, Muneta T, Ju YJ, Sekiya I (2010) Differences in kinematics of single leg squatting between anterior cruciate ligament-injured patients and healthy controls. Knee Surg Sports Traumatol Arthrosc 18: 56-63.

7. Delahunt E, Sweeney L, Chawke M, Kelleher J, Murphy K, et al. (2012) Lower limb kinematic alterations during drop vertical jumps in female athletes who have undergone anterior cruciate ligament reconstruction. J Orthop Res 30 72-78.

8. Wojtys EM, Huston LJ (1994) Neuromuscular performance in normal and anterior cruciate ligament-deficient lower extremities. Am J Sports Med 22: 89104

9. Ageberg E (2002) Consequences of a ligament injury on neuromuscular function and relevance to rehabilitation - using the anterior cruciate ligamentinjured knee as model. J Electromyogr Kinesiol 12: 205-212.

10. Kapreli E, Athanasopoulos S (2006) The anterior cruciate ligament deficiency as a model of brain plasticity. Med Hypotheses 67: 645-650.

11. Gevins A, Smith ME, McEvoy LK, Leong H, Le J (1999) Electroencephalographic imaging of higher brain function. Philos Trans R Soc Lond B Biol Sci 354: 11251133.

12. Fries $P$ (2005) A mechanism for cognitive dynamics: neuronal communication through neuronal coherence. Trends Cogn Sci 9: 474-480.

13. Baumeister J, Reinecke K, Weiss M (2008) Changed cortical activity after anterior cruciate ligament reconstruction in a joint position paradigm: an EEG study. Scand J Med Sci Sports 18: 473-484.

*Corresponding author: Jochen Baumeister, PhD, Exercise \& Brain Laboratory, Institute of Sports Medicine, University of Paderborn, Germany, Tel: 00495251 603588; Fax: 00495251 603188; E-mail: jochen@sportmed.upb.de

Received April 27, 2012; Accepted April 30, 2012; Published May 02, 2012

Citation: Baumeister J (2012) What the Brain can Tell us in Musculoskeletal Rehabilitation. J Sports Med Doping Stud 2:e106. doi:10.4172/2161-0673.1000e106

Copyright: @ 2012 Baumeister J. This is an open-access article distributed under the terms of the Creative Commons Attribution License, which permits unrestricted use, distribution, and reproduction in any medium, provided the original author and source are credited. 
Citation: Baumeister J (2012) What the Brain can Tell us in Musculoskeletal Rehabilitation. J Sports Med Doping Stud 2:e106. doi:10.4172/21610673.1000 e106

14. Kapreli E, Athanasopoulos S, Gliatis J, Papathanasiou M, Peeters R, et al (2009) Anterior cruciate ligament deficiency causes brain plasticity: a functional MRI study. Am J Sports Med 37: 2419-2426.

15. Baumeister J, Reinecke K, Schubert M, Weiss M (2011) Altered electrocortica brain activity after ACL reconstruction during force control. J Orthop Res 29 : 1383-1389.

16. Delorme A, Mullen T, Kothe C, Akalin Acar Z, Bigdely-Shamlo N, et al. (2011) EEGLAB, SIFT, NFT, BCILAB, and ERICA: new tools for advanced EEG processing. Comput Intell Neurosci 2011: 130714 